\title{
ISOTHERMIC GASIFICATION OCTAVE
}

\author{
Mario Matiauda $^{1}$, Juan B. de León Benítez ${ }^{2}$, Luis Ernesto Arteaga ${ }^{2}$, Nora Freaza ${ }^{1}$, Marta Rivero ${ }^{1}$, Carlos Yachecen $^{1}$ \\ ${ }^{1}$ Universidad Nacional de Misiones, Posadas, Argentina \\ (1) ${ }^{* 2}$ Dpto de ingeniería Química. Facultad de Química Farmacia. Universidad Central \\ (2) de Las Villas; Carretera a Camajuaní km 5 y 1/2, Santa Clara, Villa Clara, Cuba. \\ *Correspondencia autores: J.B. de León: Tel.0534221528;juanba@uclv.edu.cu, M. Matiauda: matiauda@fceqyn.unam.edu.ar; \\ N.Freaza:nfreaz@yahoo.com..ar; M.Rivero: rivero@fceqyn.unam.edu.ar
}

\begin{abstract}
Gasification is a process of thermal convertion where the solid biomass (sawdust pellets) turns into gas by partial oxidation at high temperatures. The model used is based on the theory of the two phases (bubble - emulsion) suitable for predicting experimental results in reactions that occur in fluidized beds. The model is simulated from the set of ordinary differential equations for mass balances and energy, as well, hydrodynamic studies of porosity, velocity, fraction of bubbles, with certain assumptions such as isothermal behavior of the process
\end{abstract}

Keywords: gasification-model-simulation.

\section{Gasificación Isotérmica Con Octave}

\section{RESUMEN}

La gasificación es un proceso de termoconversión donde la biomasa sólida (pellets de aserrín) se convierte en gas, a través de oxidación parcial a temperaturas altas. El modelo utilizado se basa en la teoría de las dos fases (burbuja - emulsión), adecuado para predecir resultados experimentales en reacciones que ocurren en lechos fluidizados. El modelo se simula a partir del conjunto de ecuaciones diferenciales ordinarias para los balances de masa y de energía; como así también, estudios hidrodinámicos de porosidad, velocidad, fracción de burbujas, con determinadas suposiciones como el comportamiento isotérmico del proceso.

Palabras claves: Gasificación-modelo-simulación

\section{INTRODUCCIÓN}

En general los modelos de gasificación en lecho fluidizado se distinguen en modelos termodinámicos, modelos según régimen de flujo y modelos transitorios.

El modelo simple de fase doble [1] que supone que todo el gas en exceso a la velocidad mínima de fluidización fluye en el lecho como burbujas mientras la fase emulsión permanece estacionaria en las condiciones de fluidización mínima, es de pobre ajuste experimental.

El modelo de Davidson [2], sobre la mecánica de fluidos, avanza al suponer burbujas esféricas libre de sólidos en un lecho cuyos sólidos se desplazan fuera del camino de las burbujas como un fluido viscoso y el gas fluye a través de la región densa en situación viscosa incompresible.

A partir de éste surgieron modelos en base a consideraciones de las burbujas, la posibilidad de dos o tres regiones y por lo tanto de la cantidad de parámetros, llegando al modelo K-L [3] en tres fases (burbuja, nube, emulsión), con sus parámetros en términos de una variable como el tamaño efectivo de burbujas.

Adicionalmente existen estudios sobre el llamado modelo de flujo neto [4], que se refiere a la generación neta del número de moles de gas en la fase emulsión, debido a la devolatilización y las reacciones homo y heterogéneas, 
Mario Matiauda, Juan B. de León Benítez, et al / ITEGAM-JETIA Vol.01, No 01, pp.41-47. Março, 2015.

diferenciando en tomar modelo flujo pistón en la fase burbuja y de mezcla completa en fase emulsión.

La mayoría de ellos atendieron el tratamiento del sólido carbón, recientes trabajos [5] incorporan el estudio sobre la gasificación de biomasa.

El presente trabajo tiene como fin disponer de un programa predictivo de la distribución y concentraciones de las especies productos, durante la gasificación de pellets de biomasa (aserrín de pino) en lecho fluidizado, suponiendo las fases de burbuja y emulsión, considerando las propiedades termodinámicas, de transporte e hidrodinámicas, para su ejecución en ordenador.

\section{DESARROLLO}

Modelo de gasificación

El modelo utilizado se basa en la teoría de las dos fases (burbuja - emulsión), adecuado para predecir resultados experimentales en reacciones que ocurren en lechos fluidizados (Figura 1), donde el lecho fluidizado consiste de una fase continua (emulsión), de porosidad constante, con las partículas uniformemente distribuidas en la corriente gaseosa y una gaseosa discontinua (diluída) con gases únicamente como burbujas, eventualmente canales y flujo viscoso. Todo el gas llamado en exceso del necesario para mantener la fluidización en la fase emulsión, se supone pasa a través del lecho como burbujas libres de sólidos. El flujo y densidad en la emulsión no dependen de la velocidad superficial del gas, registrándose intercambio másico y de calor entre las fases.

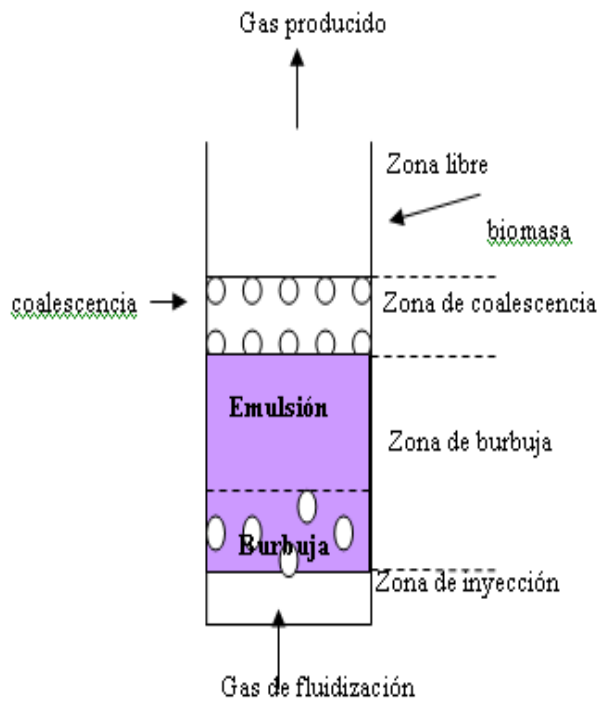

Figura.1. Zonas diferenciadas en el gasificador de LF.
Las suposiciones adoptadas se enumeran como:

- El sólido está perfectamente homogeneizado en la fase emulsión.

- Las fases de burbuja y emulsión se modelan matemáticamente para un reactor de mezcla completa (RMC)

- Los perfiles de concentración y temperatura en el gasificador en las dos fases varían en función del tiempo y no varían a lo largo del equipo.

- La biomasa está escasamente presente en la fase de emulsión, sin intercambio de calor y de masa entre el sólido y la fase de burbuja.

- El tamaño de burbuja varía con la altura del gasificador. Crecen por coalescencia y su tamaño es uniforme en cualquier sección transversal del equipo

- La transferencia de masa entre el gas y el sólido, en la fase de emulsión, es de tipo convectivo.

Se presenta un esquema simplificado del modelo bifásico en la figura 2(4).

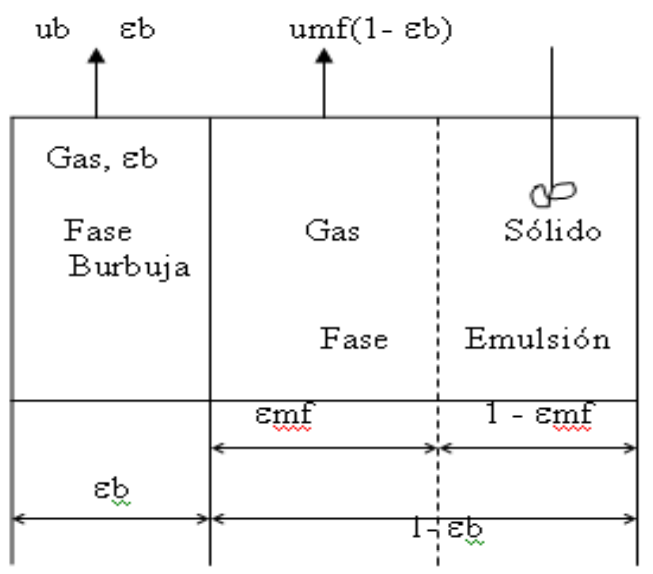

Figura 2. Modelo bifásico simplificado.

Para la simulación del modelo se incluye un conjunto de ecuaciones diferenciales ordinarias para los balances de masa y de energía; como así también, estudios hidrodinámicos de porosidad, velocidad, fracción de burbujas, número de Arquímedes.

Las ecuaciones diferenciales de balance de masa y energía están referidas a los componentes $\mathrm{C}, \mathrm{CO}, \mathrm{CO} 2, \mathrm{H} 2 \mathrm{O}, \mathrm{O} 2$, 
Mario Matiauda, Juan B. de León Benítez, et al / ITEGAM-JETIA Vol.01, No 01, pp.41-47. Março, 2015.

H2 y CH4, en las dos fases, de burbuja y de emulsión. Las ecuaciones de balance de energía refieren la temperatura en fase de burbuja y emulsión.

Las reacciones de gasificación y calores de formación a $25^{\circ} \mathrm{C}$ representativas junto a las respectivas ecuaciones cinéticas y constantes de equilibrio para el proceso de gasificación son [6]: $\mathrm{C}+\mathrm{O} 2 \rightarrow \mathrm{C} 2-408,4 \mathrm{~kJ} / \mathrm{mol}$

$\mathrm{C}+\mathrm{CO} 2 \rightarrow 2 \mathrm{CO}+172,0 \mathrm{~kJ} / \mathrm{mol}$

$\mathrm{C}+\mathrm{H} 2 \mathrm{O} \rightarrow \mathrm{CO}+\mathrm{H} 2+131,0 \mathrm{~kJ} / \mathrm{mol}$

$\mathrm{CO}+\mathrm{H} 2 \mathrm{O} \leftrightarrow \mathrm{CO} 2+\mathrm{H} 2-41,1 \mathrm{~kJ} / \mathrm{mol}$

$$
\mathrm{CH} 4+\mathrm{H} 2 \mathrm{O} \leftrightarrow \mathrm{CO}+3 \mathrm{H} 2+206,3 \mathrm{~kJ} / \mathrm{mol}
$$

Las tres primeras reacciones son heterogéneas, ocurren en la fase de emulsión; las dos últimas reacciones son homogéneas, ocurren en las fases burbuja y emulsión.

Atendiendo a las características del modelo, el gasificador de biomasa de lecho fluidizado burbujeante queda dividido en tres zonas, una de burbuja, subdividida en burbuja y emulsión, una de coalescencia o "slug" y la zona libre o "freeboard". Para el balance se consideran las dos primeras zonas; para cada componente es de rigor hacer un balance de masa en la fase de burbuja y en la de emulsión. Para el componente $\mathrm{C}$ (carbono), no existe transferencia de masa entre las fases burbuja y emulsión.

\section{Balances de masa y energía}

Balances de masa y energía en las fases1

Balance de masa.

Acumulación de masa = diferencia de flujos másicos de cada componente entre la entrada y la salida + transferencia de masa entre las fases burbuja y emulsión 7 generación/consumo de masa. (En fase burbuja) (6).

Se tiene, entonces:

$$
\frac{d y_{i}^{b}}{d t}=\frac{Q_{i}^{b}, \text { entrada }}{V} y_{i}^{b}, 0-\frac{Q_{i}^{b}, \text { salida }}{V} y_{i}^{b}+K_{i}\left(y_{i}^{e}-y_{i}^{b}\right) \pm R_{i}^{b}
$$

Resulta, entonces:

$V \rho_{m} C_{p m} \frac{d y_{14}}{d t}=\left(\sum_{i=1}^{6} F_{i}^{b}\right.$, entrada $C_{p_{i}}^{b}$, entrada $) y_{14}^{g, i}-\left(\sum_{i=1}^{6} F_{i}^{b}\right.$, salidaC ${ }_{p_{i}}^{b}$, salida $) y_{14}+f^{b} h_{b e}\left(y_{15}-y_{14}\right) \pm f^{b} \sum_{i=1}^{2}\left(\Delta H_{r_{i}}^{b} R_{i}^{b}\right) . V$

\section{Fase emulsión.}

\section{Balance de masa.}

a) Para el gas.

Acumulación de masa = diferencia de masa de gas entre la entrada y la salida+ transferencia de masa entre las dos fases \pm generación/consumo de masa por reacción. (En fase emulsión).

Entonces, se tiene:

$$
\varepsilon \frac{d y_{i}^{e}}{d t}=\frac{Q_{i}^{e}, \text { entrada }}{V} y_{i}^{e}, 0^{-}-\frac{Q_{i}^{e}, \text { salida }}{V} y_{i}^{e}+K_{i}\left(y_{i}^{b}-y_{i}^{e}\right) \pm R_{i}^{e}
$$

b) Para el sólido
Acumulación de masa del sólido = diferencia de masa del sólido entre la entrada y la salida \pm consumo de masa del sólido por reacción. (En fase emulsión). (12)

Se tiene, entonces:

$\beta \frac{d y_{13}}{d t}=\frac{Q_{\text {biomasa }} \text { entrada }}{V} y_{13,0}^{e}-\frac{Q_{\text {biomasa }} \text { salida }}{V} y_{13}^{e} \pm R_{\text {biomasa }}^{e}$

\section{Balance de energía.}

Acumulación de energía = diferencia entre la entrada y la salida de energía + calor intercambiado entre las dos fases+ calor intercambiado entre gas y sólido $\sqsupset$ calor generado por reacción. (En fase emulsión). (14). 
Resulta, entonces:

$u b=u_{0}-u m f+0,711 \sqrt{\left.g \cdot d_{b}\right)}$

Fracción de burbujas y fracción de emulsión, se calcula a partir de

$$
V \rho_{m} C_{p m} \frac{d y_{15}}{d t}=\left(\sum_{i=1}^{7} F_{i}^{e}, \text { entradaC } p_{i}^{e}, \text { entrada }\right) T_{\text {gas }}-\left(\sum_{i=1}^{7} F_{i}^{e}, \text { salidaC }{ }_{p_{i}}^{e}, \text { salida }\right) y_{15}+f^{e} h_{b e}\left(y_{14}-y_{15}\right)
$$

Las ecuaciones diferenciales ordinarias (problema de condición de frontera $y$ valor inicial) representativas asumen: a) concentraciones de los componentes valorados al tiempo inicial son iguales para fase burbuja y emulsión b) la concentración de los componentes (fase burbuja-emulsión) inicial es igual a la concentración del componente menos el flujo difusivo c) la variación de los componentes (fase burbuja-emulsión) respecto a la altura lecho es nula.

Suponiendo comportamiento isotérmico, dado el corto tiempo transitorio para alcanzar la temperatura de régimen, se desprecian las ecuaciones del balance de energía para la simulación del proceso

${ }^{1}$ Los subíndices de yi significan:1-3-5-7-9-11 corresponden a oxígeno, dióxido, monóxido,agua,hidrógeno y metano en fase burbuja, respectivamente, símil 2-6-8-10-12 en fase emulsión, 14-15 temperatura fases burbuja-emulsión, respectivamente

\section{Aspectos hidrodinámicos del modelo.}

Para el gasificador de lecho burbujeante se adoptan los parámetros hidrodinámicos, descriptos por las ecuaciones:

1. Velocidad mínima de fluidización. (8)

$$
u m f=\left(\frac{\mu_{g}}{\rho_{g} d_{p}}\right)[\sqrt{(1135,7+0,0408 A r)}-33,7]
$$

donde el número de Arquímedes, Ar, está dado por la expresión:

$$
A r=\frac{d p^{3} \rho_{g}\left(\rho_{s}-\rho_{g}\right) g}{\mu_{g}{ }^{2}}
$$

2. Velocidad de burbuja [2] $f_{b}=\frac{u_{0}-u m f}{u_{b}} ; f_{e}=1-f_{b}$

3 Velocidad de emulsión. ${ }^{(3)}$

$u_{e}=\frac{u m f}{\left(1-f_{b}\right)}$

4. Porosidad a la velocidad de fluidización mínima (9)

$$
a m f=0,4025+603,7 . d_{p}
$$

5 Porosidad en la fase de burbuja. .(3)

$$
\varepsilon_{b}=0,784-0,139 \cdot \exp \left[\frac{\left(-u_{0}-u m f\right)}{0,272}\right]
$$

6. Porosidad del lecho. (3)

$$
\varepsilon=\delta_{e} \varepsilon_{e}+\delta_{b} \varepsilon_{b}
$$

7. Coeficiente de transferencia de masa de cada componente entre las dos fases [3].$^{(3)}$

i) Entre burbuja y nube.

$$
K_{i},{ }_{b n}=4,5 \frac{u m f}{d_{p}}+5,85 \frac{\left(D_{i}^{1 / 2} \cdot g^{1 / 4}\right)}{d_{b}^{1,25}}
$$

ii) Entre nube y emulsión.

$$
K_{i, n e}=6,78\left(\frac{\varepsilon m f \cdot D_{i} u b}{d_{b}{ }^{3}}\right)^{1 / 2}
$$


Mario Matiauda, Juan B. de León Benítez, et al / ITEGAM-JETIA Vol.01, No 01, pp.41-47. Março, 2015.

iii) Entre las fases burbuja y emulsión.

$$
\frac{1}{K_{i}}=\frac{1}{\left(K_{i},{ }_{b n}\right)_{b}}+\frac{1}{\left(K_{i},_{n e}\right)_{e}}
$$

8. Coeficiente de transferencia de calor entre las fases burbuja y emulsión [3](3)

i) Entre burbuja y nube.

$$
\left(h_{b n}\right)_{b}=4,5 \frac{u m f C_{m} C_{p m}}{d_{b}}+5,85 \frac{\left(\left(C_{m} C_{p m} K_{m}\right)^{1 / 2} \cdot g^{1 / 4}\right)}{d_{b}^{1,25}}
$$

ii) Entre nube y emulsión.

$$
\left(h_{n e}\right)_{e}=6,78\left(\frac{\varepsilon m f \cdot \kappa_{m} C_{m} C_{p m} u_{b}}{d_{b}^{3}}\right)^{1 / 2}
$$

iii) Entre las fases burbuja y emulsión.

$$
\frac{1}{h_{b e}}=\frac{1}{\left(h_{b n}\right)_{b}}+\frac{1}{\left(h_{n e}\right)_{e}}
$$

\section{Programa de simulación}

El programa, bajo Octave versión 3.2.4 (para Windows) y empleo, de la interfaz gráfica de usuario GUI Octave 1.1.1. requiere el ingreso de propiedades y parámetros del modelo isotérmico, reconocidos como:

$\mathrm{R}$ (constante cinética de los gases) [J/molK], Mp (peso molecular carbono), Pp(densidad del char)(gr/m3), Emf (porosidad a fluidización mínima), dp (diámetro partícula lecho)(m), Q (caudal aire)(m3/s),V (volumen gasificador) (m3), Hbe (coeficiente de calor entre fase burbuja $y$ fase emulsión) $\left(\mathrm{kJ} / \mathrm{m} 3 \mathrm{~s}^{\circ} \mathrm{K}\right)$, Kbe (coeficiente de masa entre fase burbuja y fase emulsión) (s-1),T temperatura de régimen $\left({ }^{\circ} \mathrm{K}\right)$,tiempo de simulación(s), concentraciones iniciales de las especies(carbono, oxigeno, hidrógeno, agua)(mol/m3).

La ejecución del programa dará las concentraciones de los especies productos de la gasificación (carbono, oxígeno, hidrógeno, agua, monóxido de carbono. dióxido de carbono, metano) (mol/m3)

La resolución del modelo, sistema de ecuaciones diferenciales ordinarias se realiza con el resolvedor ode45(Runge Kutta explícito de orden 4).

\section{$\underline{\text { Resultados }}$}

Para la simulación computacional del modelo matemático, se presentan los resultados para una selección particular de los parámetros (valores característicos del proceso de gasificación para la materia prima aserrín de pino):

Flujo de aserrín: $015 \mathrm{~kg} / \mathrm{h}$ (pellets y a granel), de fórmula empírica C4.2 H6.1 O2.7

Humedad del aserrín: inferior a 6\%; caudal de aire: $2 \mathrm{~m} 3 / \mathrm{h}$ (a Patm); $\mathrm{T}$ temperatura de operación reactor: $800^{\circ} \mathrm{C}$; porosidad:0.55; $\mathrm{dp}=0.0005 \mathrm{~m} ; \mathrm{Kbe}=22 \mathrm{~s}-1 ; \mathrm{V}=0.00113 \mathrm{~m} 3 ;$ tiempo simulación: $10 \mathrm{~s}$

Se presentan las gráficas de concentración de hidrógeno, en función del tiempo, como el conjunto de las especies gaseosas (figuras1 y 2)

\section{CONCENTRACIONESFNALES}
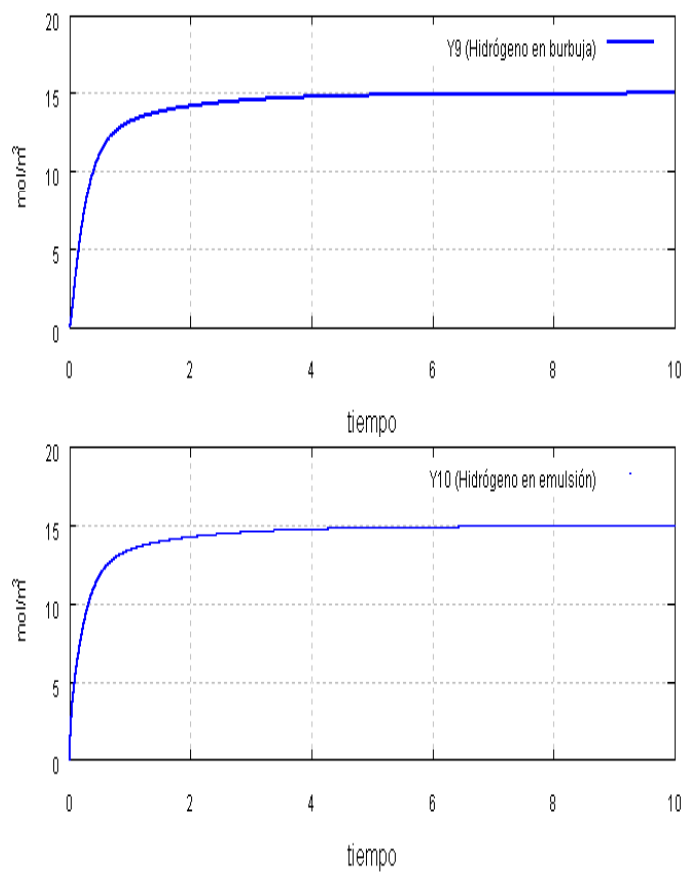

Figura 3- Concentración de hidrogeno

\section{CONCLUSIONES}

El modelo, en referencia a la concentraciones finales de las especies gaseosas, muestra significativa influencia de la concentración inicial de carbono (flujo másico de biomasa y composición de la biomasa) y de agua (contenido de humedad de la biomasa, posibilidad de inyección secundaria de vapor o mezclas aire-vapor como comburentes), principalmente 
Mario Matiauda, Juan B. de León Benítez, et al / ITEGAM-JETIA Vol.01, No 01, pp.41-47. Março, 2015.

traducidas en el consumo pleno del carbono y concentraciones finales altas de hidrógeno y descenso de monóxido.

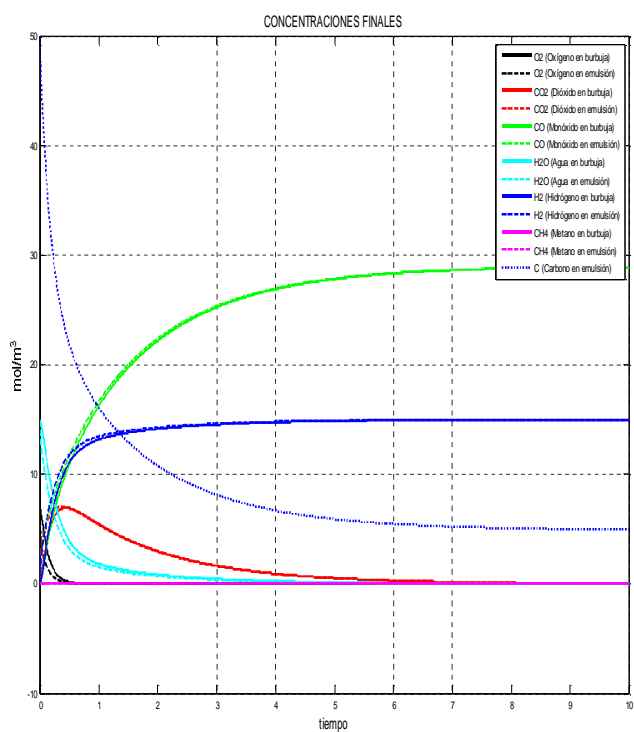

Figura 4 - Concentraciones de especies gaseosas

La variación del nivel de temperaturas en el intervalo usual para los procesos de gasificación $\left(800-1000^{\circ} \mathrm{C}\right)$ no ejerce importante significado sobre las concentraciones finales. En el mismo sentido se manifiesta la variación del caudal de aire.

Registra influencia la condición del lecho, es decir valores más altos de porosidad son proporcionales a la disminución de concentración final de carbono y al aumento del contenido de hidrógeno.

Para la simplificación del modelo se han tomado valores constantes del coeficiente de transferencia de masa, su variación como parámetro no incide significativamente en las concentraciones finales de las especies gaseosas.

En definitiva, se manifiesta un significativo control del proceso por las reacciones involucradas, a través de su expresión cinética, de cualquier manera de origen empírico.

El modelo simulado, basado en una teoría simplificada del fenómeno de gasificación, deja abierto el estudio más exhaustivo de variables y parámetros, avanzando sobre el grado de complejidad, que junto a las prácticas experimentales en el contexto de la técnica de lecho fluidizado, permitirá enriquecer los aportes de la investigación en este campo y en esta escala, incluyendo las herramientas matemáticas empleadas.

\section{NOMENCLATURA}

$a_{s}=$ difusividad térmica $\left(\mathrm{m}^{2} / \mathrm{s}\right)$

Ar: Número de Arquímedes (adim)

$C_{p_{i}}^{e}=$ capacidad calorífica de cada componente en fase emulsión $\left(\mathrm{J} / \mathrm{kmol}^{\circ} \mathrm{K}\right)$

$C_{p_{i}}^{b}=$ capacidad calorífica de cada componente en fase burbuja $\left(\mathrm{J} / \mathrm{kmol}^{\mathrm{o}} \mathrm{K}\right)$

$C_{p m}$ = capacidad calorífica de la mezcla $\left(\mathrm{J} / \mathrm{kmol}^{\circ} \mathrm{K}\right)$

$C_{m}=$ concentración mezcla gasesosa $\left(\mathrm{kmol} / \mathrm{m}^{3}\right)$

$d_{p}=$ diámetro de partícula del lecho $(\mathrm{m})$

$d_{b}=$ diámetro de burbuja $(\mathrm{m})$

$D_{i}=$ coeficiente difusivo $\left(\mathrm{m}^{2} / \mathrm{s}\right)$

$f_{b}=$ fracción de burbuja (adim)

$f_{e}=$ fracción de emulsión (adim)

$F_{i}=$ flujo másico del componente $i(\mathrm{~kg} / \mathrm{s})$

$g=$ aceleración de la gravedad $\left(\mathrm{m} / \mathrm{s}^{2}\right)$

$h_{b e}=$ coeficiente de calor entre fases burbuja y emulsión $\left(\mathrm{kJ} / \mathrm{m}^{3} \mathrm{~s}{ }^{\mathrm{o}} \mathrm{K}\right)$

$\left(h_{b n}\right)_{b}=$ coeficiente de calor entre fases burbuja- nube, fase burbuja $\left(\mathrm{kJ} / \mathrm{m}^{3} \mathrm{~s}{ }^{\circ} \mathrm{K}\right)$

$\left(h_{n e}\right)_{e}=$ coeficiente de calor entre nube y emulsión, en fase emulsión $\left(\mathrm{kJ} / \mathrm{m}^{3} \mathrm{~s}^{\mathrm{O}} \mathrm{K}\right)$.

$K_{i}=$ coeficiente de masa para el componente $i\left(\mathrm{~s}^{-1}\right)$

$K_{i},{ }_{b n}=$ coeficiente de masa para el componente $i$, entre burbuja y nube $\left(\mathrm{s}^{-1}\right)$.

$K_{i},{ }_{n e}=$ coeficiente de masa para el componente $i$, entre nube y emulsión $\left(\mathrm{s}^{-1}\right)$.

$\left(K_{i},{ }_{b n}\right)_{b}=$ coef. masa para el componente $i$, entre burbuja y nube, en fase burbuja $\left(\mathrm{s}^{-1}\right)$

$\left(K_{i},{ }_{n e}\right)_{e}=$ coef. masa para el comp. $i$, entre nube y emulsión, en fase emulsión $\left(\mathrm{s}^{-1}\right)$

$k_{g}{ }^{e}=$ conductividad térmica de la mezcla en la emulsión $\left(\mathrm{kJ} / \mathrm{m}^{\circ} \mathrm{K}\right)$

$L=$ longitud del pellet (m)

$M=$ masa del pellet $(\mathrm{g})$ 
Mario Matiauda, Juan B. de León Benítez, et al / ITEGAM-JETIA Vol.01, No 01, pp.41-47. Março, 2015.

$Q_{i}^{b}=$ caudal fase burbuja $\left(\mathrm{m}^{3} / \mathrm{s}\right)$

$Q_{i}^{e}=$ caudal fase emulsión $\left(\mathrm{m}^{3} / \mathrm{s}\right)$

$Q_{\text {biomasa }}=$ caudal $\left(\mathrm{m}^{3} / \mathrm{s}\right)$

$u b=$ velocidad en fase burbuja $(\mathrm{m} / \mathrm{s})$

$u_{e}=$ velocidad en fase emulsión $(\mathrm{m} / \mathrm{s})$

$u m f=$ velocidad de fluidización mínima $(\mathrm{m} / \mathrm{s})$

$u_{0}=\operatorname{velocidad}$ inicial $(\mathrm{m} / \mathrm{s})$

$t_{v}=$ tiempo de devolatilización (s)

$t_{c}=$ tiempo de combustión del char (s)

$V=$ volumen gasif. $\left(\mathrm{m}^{3}\right)$

$R_{i}^{b}=$ generación/consumo de componente $i$ en fase burbuja $\left(\mathrm{mol} / \mathrm{m}^{3} \mathrm{~s}\right)$

$R_{i}^{e}=$ generación/consumo de componente $i$ en fase emulsión $\left(\mathrm{mol} / \mathrm{m}^{3} \mathrm{~s}\right)$

$y_{i}^{b}=$ concentración de componente $i$ en fase burbuja $(i=1,3,5,7,9,11,13)\left(\mathrm{mol} / \mathrm{m}^{3}\right)$

$y_{i}^{0}=$ concentración inicial de componente $i\left(\mathrm{~mol} / \mathrm{m}^{3}\right)$.

$y_{i}^{e}=$ concentración de componente $i$ en fase emulsión $(i=2,4,6,8,10,14)\left(\mathrm{mol} / \mathrm{m}^{3}\right)$.

$y_{14}^{e}, y_{15}^{b}=$ temperaturas de emulsión y burbuja, respectivamente $\left({ }^{\circ} \mathrm{K}\right)$

$\delta=$ fracción de volumen en cada fase (adim)

$\varepsilon=$ porosidad del lecho (adim), $\varepsilon_{b}=$ porosidad fase burbuja (adim), $\varepsilon_{e}=$ porosidad fase emulsión (adim)

$\kappa=$ conductividad térmica mezcla $\left(\mathrm{kJ} / \mathrm{ms}^{\circ} \mathrm{K}\right)$

$\rho_{g}=$ densidad del gas $\left(\mathrm{kg} / \mathrm{m}^{3}\right)$

$\rho_{m}=$ densidad de la mezcla gaseosa $\left(\mathrm{kg} / \mathrm{m}^{3}\right)$

$\rho_{s}=$ densidad del sólido $\left(\mathrm{kg} / \mathrm{m}^{3}\right)$

$\mu_{g}=$ viscosidad del gas $(\mathrm{kg} / \mathrm{ms})$

$\phi=$ diámetro del pellet $(\mathrm{mm})$

$\Delta H_{r_{i}=\text { entalpía de reacción en fase emulsión }(\mathrm{J} / \mathrm{kmol})}^{e}$

$\Delta H_{r_{i}=\text { entalpía de reacción en fase burbuja }(\mathrm{J} / \mathrm{kmol})}^{b}$

$\mathrm{R}=$ Constante cinética de los gases $[\mathrm{J} / \mathrm{molK}]$

\section{REFERENCIAS BIBLIOGRÁFICAS}

[1] Toomey, R.D; Johnstone, H.F.; Gas fluidization of solid particles, Chem.Eng.Prog, 48, 220, 1953.

[2] J.F. Davidson, D. Harrison, Fluidized Particles, Cambridge Univ.Press, New York, 1963.

[3] Kunii, D, Levenspiel, O.-Fluidization engineering-2nd edition, Butterworth-Heinemann.

[4] Yan,H.M, Heidenreich,C., Zhang,D.; Mathematical modeling of a bubbling fluidized-bed coal gasifier and the significance of net flow-Fuel. Vol. 77, No. 9/10, pp. 1067-1079, 1998

[4] Yang,Y.B., Ryu,C.,Khor, A.,Yates,N.E., Sharifl, B:N:, Swithenbank, J.;Effect of fuel properties on biomass combustion. Part II. Modeling approach-identification of the controlling factors-Fuel 84, 2116-2130,2005

[5] Petersen, I., Werther, J., Experimental investigation and modelling of gasification of sewage sludge in the circulating fluidized bed. Chemical Engineering and Processing, 44, 717736,2005

[6] Sadaka, S.S., Ghaly, A.E., Sabbah, M.A.; Two phases biomass-air-steam gasification model for fluidized bed reactors: part I-model development; Biomass \& Bioenergy, 22, 439-462, 2002.

[7] Botterill,J.S.M.,Bessant,D.J.,The flow properties of fluidized solids; Powder Technology,14,(131),1976

[8] Abrahamsen, A.R, Geldart, D., Behavior of Gas-Fluidized Beds of Fine Powders. Part I, Homogeneous luidization;Powder Technology,29,3546,pp 